\title{
An item-oriented recommendation algorithm on cold-start problem
}

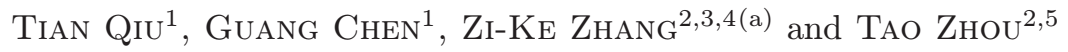 \\ ${ }^{1}$ School of Information Engineering, Nanchang Hangkong University - Nanchang 330063, PRC \\ ${ }^{2}$ Web Sciences Center, University of Electronic Science and Technology of China - Chengdu 610054, PRC \\ ${ }^{3}$ Institute of Information Economy, Hangzhou Normal University - Hangzhou 310036, PRC \\ ${ }^{4}$ Department of Physics, University of Fribourg - Chemin du Musée, 1700 Fribourg, Switzerland \\ ${ }^{5}$ Department of Modern Physics, University of Science and Technology of China - Hefei 230026, PRC
}

\begin{abstract}
Based on a hybrid algorithm incorporating the heat conduction and probability spreading processes (Proc. Natl. Acad. Sci. U.S.A., 107 (2010) 4511), in this letter, we propose an improved method by introducing an item-oriented function, focusing on solving the dilemma of the recommendation accuracy between the cold and popular items. Differently from previous works, the present algorithm does not require any additional information (e.g., tags). Further experimental results obtained in three real datasets, RYM, Netflix and MovieLens, show that, compared with the original hybrid method, the proposed algorithm significantly enhances the recommendation accuracy of the cold items, while it keeps the recommendation accuracy of the overall and the popular items. This work might shed some light on both understanding and designing effective methods for long-tailed online applications of recommender systems.
\end{abstract}

Introduction. - A huge amount of data rushes into our everyday life, which traps us in the difficulty of deciding what we actually choose. For example, picking up a preferable TV program to watch from too many programs, or getting desirable goods when confronting multifarious commodities [1]. How to sort useful information out of the information sea is becoming one of the central research topics. Recommender system emerges as an important tool to make predictions for the possible future interests of users according to their historical activities [2]. With the personalized recommendation inquiry, different algorithms have been proposed, such as the collaborative filtering [3], the content-based method [4], as well as their relevant extensive studies [5-10].

Recently, thanks to fruitful achievements of complexity theory, recommendation algorithms based on complex networks have attracted an increasing attention from both computer science and the physical community [11-19]. In particular, the concepts of heat conduction $[11,16]$ and mass diffusion $[12,16]$ in traditional physical domain are incorporated into recommender systems, and provide a

\footnotetext{
(a) E-mail: zhangzike@gmail.com
}

promising way to probe a new generation of recommender systems from the perspective of physics. Inspired by these works, a variety of physical-concept-based methods have been proposed, and achieved a significant success in personalized recommendations. For example, the attempt in solving the apparent dilemma between accuracy and diversity by an elegant hybridization of heat conduction and probability spreading algorithms in [16].

Whereas investigating the recommendation accuracy from the overall system point of view, recommendation focusing on users or items with little information still remains challenging, which also refers to the so-called cold-start problem. For the newly added or the relatively inactive users or unpopular items, it is difficult to make predictions due to the lackness of activity records. It is worth noting that such kind of users or items usually occupy a large proportion of the overall users or items in most online applications. Related works concerning this issue include the heuristic similarity measurements [20], and the social-tag-based algorithms $[18,19,21]$.

The key challenge of cold-start problem is that focusing strongly on the recommendation accuracy of the cold items, while putting the accuracy of the overall or the 
popular items at risk. Apparently, popular items are widely accepted by users. Therefore, it is essential to keep the recommendation accuracy of the popular items when improving the recommendation accuracy of the cold items.

In this letter, we show how this dilemma is resolved by introducing an item-degree-dependent parameter in a hybrid method. Based on three real datasets, $R Y M$, Netflix and MovieLens, we show that, while keeping the recommendation accuracy of the popular and the overall items, the recommendation accuracy of the cold items are significantly improved by appropriately tuning the itemdegree-dependent parameter. Compared with a relevant study of solving the cold-start problem by introducing social tags [21], the proposed algorithm does not require any additional information, but directly performs on the bipartite network.

Algorithm. - A recommender system can be characterized by a bipartite graph composed of users and items, with the user set labeled as $U=\left\{u_{1}, u_{2}, \ldots, u_{m}\right\}$ and the item set labeled as $O=\left\{o_{1}, o_{2}, \ldots, o_{n}\right\}$. The relation between users and items can be formulated by an adjacent matrix $A$, with $a_{\alpha i}=1$ for the linked user-item pairs, and $a_{\alpha i}=0$ for the unconnected pairs. Namely, popularity of an item can be described by the item degree, $k_{\alpha}$. Thus, items which take larger value of degrees are regarded as the popular items, and which have smaller value of degrees are considered as the cold items.

We begin with the hybrid method of heat conduction and probability spreading (HHP) proposed in [16], which shows a high efficiency in both the accuracy and the diversity of recommendation. If we assign each item an initial level of "resource" labeled by a vector $\overrightarrow{f_{0}}=\left(f_{1,0}^{i}, \ldots, f_{n, 0}^{i}\right)$, the final resource of the item $\vec{f}=\left(f_{1}^{i}, \ldots, f_{n}^{i}\right)$ is obtained according to a reallocation process, which can be described in a transformation form,

$$
\vec{f}=W \overrightarrow{f_{0}}
$$

where $W$ is the transformation matrix of resource reallocation. By ranking the level of final resources, items with higher resources will be recommended to users. Therefore, how to define the transformation matrix, $W$, plays a key role in the recommendation process.

In the heat conduction algorithm, resource allocation is an averaging process. At first, users receive an average level resources from their neighboring items, and then the items again get a feedback of the average resource from its neighboring users. By this heat-conducting analogous resource reallocation process, the items with smaller degrees will be promoted to have the chance to obtain more resources, with the transformation matrix formulated by

$$
W_{\alpha \beta}^{H}=\frac{1}{k_{\alpha}} \sum_{j \in U} \frac{a_{\alpha j} a_{\beta j}}{k_{j}},
$$

where $k_{\alpha}$ is the degree of the item $o_{\alpha}$, and $k_{j}$ is the degree of the user $u_{j}$. The heat conduction algorithm shows a good performance in recommendation diversity [16], however, at the cost of recommendation accuracy for assigning more resources on cold items.

On the other hand, in the probability spreading algorithm, the item firstly distributes the resource to its neighboring users with an equal probability, while the user again redistributes its total level of resource to its neighboring items. The item then obtains its final level of resource by summing up all the resources from its neighboring users. Therefore, the items with a relatively large number of links will obtain more resources, with the transformation matrix formulated by

$$
W_{\alpha \beta}^{P}=\frac{1}{k_{\beta}} \sum_{j \in U} \frac{a_{\alpha j} a_{\beta j}}{k_{j}},
$$

where $k_{\beta}$ is the degree of the item $o_{\beta}$. The probability spreading algorithm focuses more on the popular items, and therefore shows a high recommendation accuracy, which, however, potentially puts the recommendation diversity at risk.

In order to take the advantages of the aforementioned methods, the HHP method [16] was proposed to partially solve the dilemma between the accuracy and diversity of recommendation by elegantly combining the heat conduction and probability spreading method as

$$
W_{\alpha \beta}^{H+P}=\frac{1}{k_{\alpha}^{1-\lambda} k_{\beta}^{\lambda}} \sum_{j \in U} \frac{a_{\alpha j} a_{\beta j}}{k_{j}} ;
$$

when tuning the hybridization parameter, $\lambda \in[0,1]$, to a suitable value, the HHP method outperforms in both accuracy and diversity of recommendation with respect to the heat conduction (HeatS) and the probability spreading (ProbS) methods.

In order to further investigate the effects of $\lambda$ on recommendation, in fig. 1 we show the average degree of recommended items in the top $L$ recommendation list in the phase diagram of $(\lambda, L)$ for Netflix. One observes a positive relation between $\lambda$ and the average degree, i.e., on average, the system prefers to recommend lowdegree items for small value of $\lambda$, and recommend highdegree items for large value of $\lambda$. In [16], the system achieves the optimal balance of recommendation for the cold items and the popular items at $\lambda=0.23$ for Netflix. When we investigate the item degree distribution (see fig. 1), we find a broad range of distribution with a power-law like, where the cold items occupy a large proportion. Similar phenomena are also observed in $R Y M$ and MovieLens. This inspires us an intuition that, if we assign each item a different value of $\lambda$ according to its own degree, the system might be more efficient in recommending heterogenous items, hence enhance the recommendation capability for the cold items. Therefore, to better understand the effects of $\lambda$ in eq. (4), we analytically investigate the recommendation bias for the hybrid algorithm. On average, the probability for a target user $i$ collecting item $\beta$, is directly proportional to $\beta$ 's degree, 

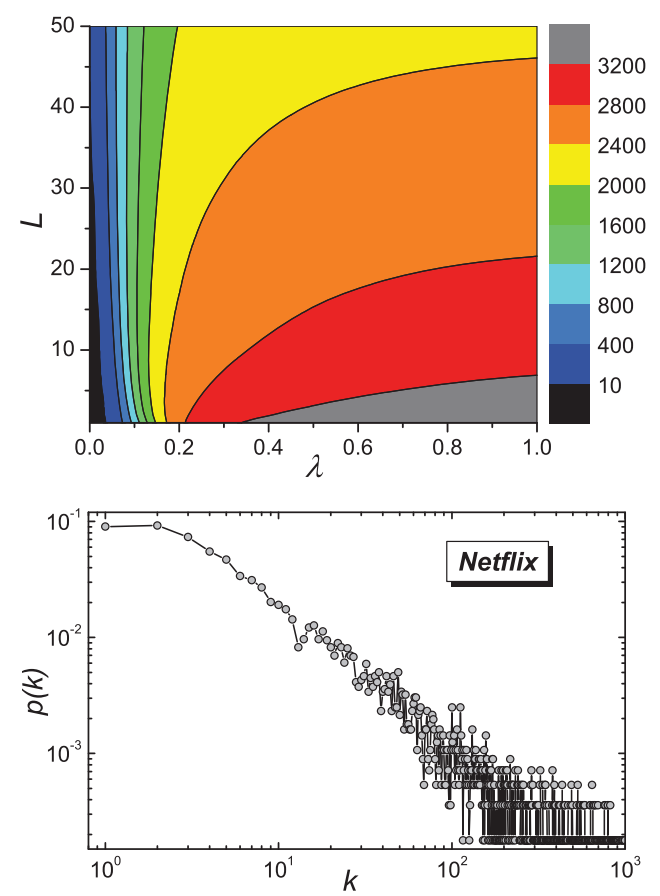

Fig. 1: (Color online) In the upper panel, the average degree of recommended items in the top $L$ recommendation list is displayed in the $(\lambda, L)$ space with different colors for the Netflix. The border separates the lower degree and the higher degree, which makes up a phase boundary. The lower panel shows the item degree distribution.

that is to say, $a_{i \beta} \propto \frac{k_{\beta}}{n}$, where $n$ is the number of items. We then hypothesize the probability that $a_{i \beta}$ is independent of other links, and the expected score of each user-item link, $f_{i \alpha}$, can be calculated as

$$
\begin{aligned}
f_{i \alpha} & =\sum_{\beta} W_{\alpha \beta}^{H+P} a_{i \beta} \\
& =\sum_{\beta} \frac{a_{i \beta}}{k_{\alpha}^{1-\lambda} k_{\beta}^{\lambda}} \sum_{j \in U} \frac{a_{\alpha j} a_{\beta j}}{k_{j}} \\
& \propto \sum_{\beta} \frac{k_{\beta}}{k_{\alpha}^{1-\lambda} k_{\beta}^{\lambda}} \sum_{j \in U} \frac{k_{\alpha} k_{\beta}}{k_{j}} \\
& \propto k_{\alpha}^{\lambda} \sum_{\beta} k_{\beta}^{2-\lambda} .
\end{aligned}
$$

Since each user has his/her own activity, to properly evaluate the optimal $\lambda$ for the whole effect, we rescale $f_{i \alpha}$ to reflect the heterogeneous effect by setting $f_{i \alpha}=\frac{f_{i \alpha}}{k_{i}}$. Figure 2 shows the numerical results of seeking for the optimal $\lambda$ in three representive datasets. One can see from fig. 2 that, in all the three experiments, optimal results reach at relatively small $\lambda$, which suggests that HeatS plays a more important role in the hybrid case. Thus, items with small degree might improve the overall recommendation performance. It further suggests that assigning diverse $\lambda$ for different items would help to find better recommendation results.

With this incentive, we propose an item-oriented HHP algorithm (OHHP) by replacing the original unique $\lambda$ for

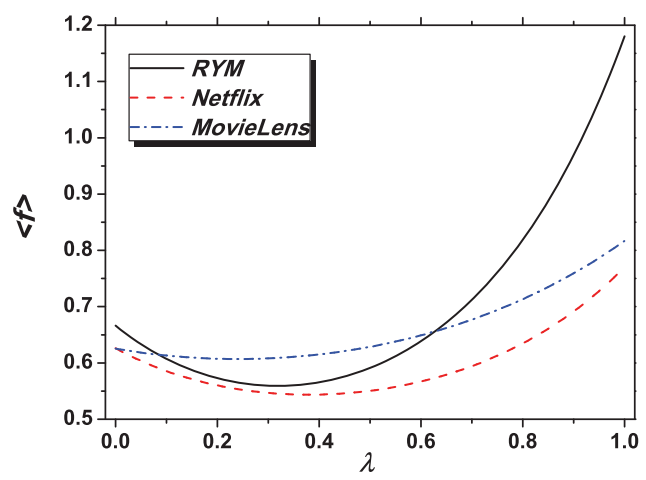

Fig. 2: (Color online) Numerical results for approaching the optimal $\lambda$ for the three datasets.

all items by a function form positively related to item degree,

$$
\lambda=\left(\frac{k_{\beta}}{k_{\max }}\right)^{\gamma},
$$

where $k_{\beta}$ is the degree of the examined item, $k_{\max }$ is the maximum degree of all the items, and $\gamma$ is a tuned parameter. By incorporating the item-degree-dependent hybridization parameter, $\lambda$, the probability spreading factor in the transformation matrix of eq. (4) can be optimized for different degree level items in recommendation accuracy. Moreover, compared with a previous study which successfully resolved the cold-start problem, they employed tags as accessorial information [22], whereas the present algorithm only uses the bipartite graph structure by merely transforming the parameter from $\lambda$ to the $k_{\beta^{-}}$ dependent function with a tunable parameter $\gamma$. Therefore, it provides a simpler way to design effective methods for online applications, especially when lacking accessorial information.

To provide more comprehensive comparisons, we additionally employ a widely adopted recommendation technique, user-based collaborative filtering $(\mathrm{CF})$ method, to recommend items for a given user by calculating the similarity between users $i$ and $j$ via a cosine similarity $s_{i j}=\frac{\sum_{\alpha} a_{\alpha i} a_{\alpha j}}{\sqrt{k_{i} k_{j}}}$. If an item has not been collected by user $i$, s/he will score item $\alpha$ by summarizing all the users similar with him/her, $f_{\alpha i}=\sum_{j \neq i} s_{i j} a_{\alpha j}$. Then the final recommendation list will be made for user $i$ according to the descending order of the recommendation score $f_{\alpha i}$.

Data. - The datasets we analyzed are based on the $R Y M$, the Netfilx and the MovieLens. The RYM and Netflix are provided by [16]. The $R Y M$ is a music rating system with a ten-level rating, and the Netflix and MovieLens are movie rating systems with a five-level rating. The $R Y M$ dataset is downloaded from the music rating web site RateYourMusic.com, the Netflix dataset is obtained by randomly selecting from the huge dataset of the Netflix Prize, and the MovieLens is downloaded from the web site of GroupLens Research ${ }^{1}$. In addition,

\footnotetext{
${ }^{1}$ http://grouplens.org/.
} 
Table 1: Basic statistics of the RYM, the Netflix and the MovieLens. $m, n, e, s$ denote the number of users, items, links, and the sparsity, respectively.

\begin{tabular}{ccccc}
\hline Dataset & $m$ & $n$ & $e$ & $s$ \\
\hline RYM & 33786 & 5381 & 613387 & $3.37 \%$ \\
Netflix & 10000 & 6000 & 701947 & $1.17 \%$ \\
MovieLens & 943 & 1682 & 82520 & $5.20 \%$ \\
\hline
\end{tabular}

we perform a coarse-graining mapping to the unary form for all the three datasets. That is to say, we keep the links if their respective ratings are no less than three for the Netflix and the MovieLens, and six for the RYM. The sparsity of the datasets is defined as the total number of links proportional to the all possible pairs of the useritem links. Table 1 summarizes the basic statistics of the purified three datasets.

To test our algorithm performance, we divide the total links between users and items into two subsets: the training set and the test set. The training set is used to make predictions, and the test set is used to test the performance of algorithms. We randomly remove $10 \%$ links from the total links, with the remaining 90\% links for training and the removed $10 \%$ links for testing.

Metrics. - To give solid and comprehensive evaluation of the present algorithm, we then investigate the widely adopted recommendation accuracy measure including the ranking score $(R S)$, the recall $(R)$, and the recommendation precision $(P)$.

1) Ranking score $(R S)$ [12]. The $R S$ for item $\alpha$ recommended to user $i$ is defined by $R S_{\alpha i}=\frac{p_{\alpha}}{n-k_{i}}$, where $n$ is the number of items, $k_{i}$ is the degree of user $i$, and $p_{\alpha}$ is the position of the recommended items $\alpha$ located in all the uncollected items. We assume that the items collected by a user are what the user preferred. Therefore, the deleted links in the testing set should be ranked at a higher position for an accurate recommendation. The smaller the $R S$ is, the more accurate the algorithm will be. The average ranking score $\langle R S\rangle$ is then given by averaging over deleted links in the test set.

2) Recall $(R)$ [22]. The recall $R$ is defined as $R=$ $\frac{1}{m} \sum_{i}^{m} \frac{q_{i L}}{l_{i}}$, where $q_{i L}$ is the number of user $u_{i}$ 's deleted links contained in the top $L$ recommended items list, $l_{i}$ is the number of user $u_{i}$ 's deleted links in the test set.

3) Precision $(P)[22]$. The recommendation precision $P$ is defined as $P=\frac{1}{m} \frac{\sum_{i}^{m} q_{i L}}{L}$, where $q_{i L}$ is the number of user $u_{i}$ 's deleted links contained in the top $L$ recommended items list.

Results. - The accuracy is one of the most important indicators to evaluate recommendation systems. By introducing the item-degree parameter $\gamma$, one expects an optimal value to minimize the recommendation accuracy. The overall ranking score $\langle R S\rangle$ on $\gamma$ is shown in fig. 2, where one can obtain an optimal $\gamma=0.45,1.28$, and 1.49 for the

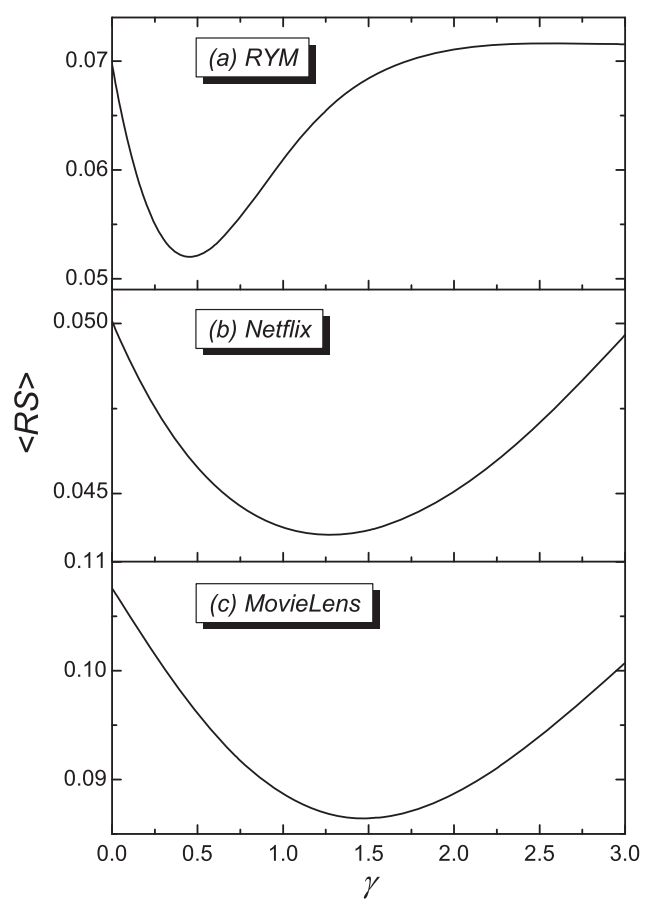

Fig. 3: The overall ranking score $\langle R S\rangle$ vs. the item-degree parameter $\gamma$ is displayed for the $R Y M$, the Netflix, and the MovieLens, respectively.

$R Y M$, the Netflix and the MovieLens, respectively. Therefore, in the following, we will emphasize on discussing the recommendation performance at these optimal values in order to compare the results with those based on HHP.

To show how the ranking score performs on items with different value of degrees, we additionally investigate an item-degree-dependent ranking score $\langle R S\rangle_{k}[23]$. The $\langle R S\rangle_{k}$ is defined as the average ranking score over items with the same value of degrees. In fig. 3, the $\langle R S\rangle_{k}$ $v s$. the item degree $k$ is displayed for the $R Y M$, the Netflix, and the MovieLens at the optimal hybridization parameters, with $\lambda=0.41$ and $\gamma=0.45$ for the RYM, $\lambda=0.23$ and $\gamma=1.28$ for the Netflix, $\lambda=0.17$ and $\gamma=$ 1.49 for the MovieLens, respectively. It is observed that, whereas stabilizing the recommendation accuracy of the popular items with large degrees, the $\langle R S\rangle_{k}$ of the OHHP method for the items with small degree value is much smaller than that in the HHP, which indicates a significant enhancement of recommendation accuracy for cold items. Compared with the CF method, the OHHP method presents a much higher recommendation accuracy for the overall items with both small and large degrees.

In traditional recommendation systems, it is difficult for users to realize the cold items with little recommendation record. However, statistics for items whose degree is no larger than 10 shows $24.51 \%$ for the $R Y M, 50.62 \%$ for the Netflix, and $41.26 \%$ for the MovieLens, i.e., cold items actually occupy a big fraction for most datasets. It essentially requires improved algorithms to make predictions for the cold items efficiently. A summary of recommendation 
Table 2: The item-degree-dependent ranking score $\langle R S\rangle_{k \leqslant 10}$, recall $R_{k \leqslant 10}$, precision $P_{k \leqslant 10}$, the overall ranking score $\langle R S\rangle$, recall $R$, precision $P$, and the item-degree-dependent ranking score $\langle R S\rangle_{k>10}$, recall $R_{k>10}$, precision $P_{k>10}$ based on the CF, the HHP and the OHHP methods are shown for the RYM, the Netflix, and the MovieLens, with $L=50$. The entries corresponding to the higher accuracies are emphasized by black.

\begin{tabular}{ccccccccccc}
\hline & & $\langle R S\rangle_{k \leqslant 10}$ & $R_{k \leqslant 10}$ & $P_{k \leqslant 10}$ & $\langle R S\rangle$ & $\langle R S\rangle_{k>10}$ & $R$ & $R_{k>10}$ & $P$ & $P_{k>10}$ \\
\hline \multirow{5}{*}{$R Y M$} & $\mathrm{CF}$ & 0.620 & 0.002 & 0.00000 & 0.094 & 0.087 & 0.509 & 0.511 & 0.030 & 0.030 \\
\cline { 2 - 10 } Netflix & $\mathrm{HHP}$ & 0.321 & 0.072 & 0.00006 & 0.055 & 0.052 & 0.622 & 0.625 & 0.041 & 0.041 \\
& $\mathrm{OHHP}$ & $\mathbf{0 . 2 1 7}$ & $\mathbf{0 . 1 6 4}$ & $\mathbf{0 . 0 0 0 1 6}$ & $\mathbf{0 . 0 5 1}$ & $\mathbf{0 . 0 4 9}$ & $\mathbf{0 . 6 2 5}$ & $\mathbf{0 . 6 2 7}$ & $\mathbf{0 . 0 4 2}$ & $\mathbf{0 . 0 4 2}$ \\
\cline { 2 - 11 } & $\mathrm{CF}$ & 0.578 & 0.000 & 0.00000 & 0.057 & 0.048 & 0.405 & 0.411 & 0.053 & 0.053 \\
\cline { 2 - 10 } & $\mathrm{HHP}$ & 0.428 & 0.009 & 0.00004 & 0.044 & 0.037 & $\mathbf{0 . 4 7 4}$ & $\mathbf{0 . 4 8 1}$ & $\mathbf{0 . 0 6 2}$ & $\mathbf{0 . 0 6 2}$ \\
\cline { 2 - 10 } & $\mathrm{OHHP}$ & $\mathbf{0 . 3 4 5}$ & $\mathbf{0 . 0 1 9}$ & $\mathbf{0 . 0 0 0 0 9}$ & $\mathbf{0 . 0 4 3}$ & 0.037 & 0.438 & 0.444 & 0.059 & 0.059 \\
\cline { 2 - 10 } & $\mathrm{CF}$ & 0.610 & 0.000 & 0.00000 & 0.116 & 0.098 & 0.438 & 0.449 & 0.070 & 0.070 \\
\cline { 2 - 10 } & $\mathrm{HHP}$ & 0.427 & 0.036 & 0.00020 & 0.085 & $\mathbf{0 . 0 7 3}$ & $\mathbf{0 . 5 3 1}$ & $\mathbf{0 . 5 4 4}$ & $\mathbf{0 . 0 8 7}$ & $\mathbf{0 . 0 8 6}$ \\
\cline { 2 - 9 } & $\mathrm{OHHP}$ & $\mathbf{0 . 3 8 5}$ & $\mathbf{0 . 0 6 3}$ & $\mathbf{0 . 0 0 0 3 7}$ & 0.085 & 0.074 & 0.528 & 0.539 & 0.085 & 0.085 \\
\hline
\end{tabular}

performance respectively given by the $\mathrm{CF}$ method, the HHP and the OHHP methods at optimal values of $\lambda$ and $\gamma$ is shown in table 2. We find that the OHHP method outperforms the CF method in all the three recommendation accuracy indicators, the $\langle R S\rangle_{k \leqslant 10}$, the $\langle R S\rangle$ and the $\langle R S\rangle_{k>10}$. More importanly, compared with the HHP method, the $\langle R S\rangle_{k \leqslant 10}$ performed by the OHHP shows a much smaller value. When looking into the recommendation accuracy of the overall and the popular items, both $\langle R S\rangle$ and $\langle R S\rangle_{k>10}$ show even a little enhancement for the $R Y M$, and remain statistically steady for the Netflix and the MovieLens. To better understand the recommendation performance, we show the degree distribution $p(k)$ for the items in the top $L=50$ recommendation list for the RYM, the Netflix, and the MovieLens in fig. 4, where one can clearly observe that the probability of the cold items by the OHHP method is larger than that by the HHP method, while by the CF method is almost zero. We then argue that the OHHP method might be effective for cold-items recommendation.

Further investigation on the overall recall, $R$, are presented to be much improved for all the three datasets in comparison with the CF method, and a little enhanced for the $R Y M$, and stabilized for the Netflix and the MovieLens in comparison with the HHP method. To focus on the recall performance specially on the cold items, we analogously introduce an item-degree-dependent recall measure which reads $R_{k}=\frac{1}{m} \sum_{i}^{m} \frac{q_{i L}^{k}}{l_{i}^{k}}$, where $q_{i L}^{k}$ is the number of user $u_{i}$ 's deleted links for items with degree $k$ in the top $L$ recommended items list, and $l_{i}^{k}$ is the number of user $u_{i}$ 's deleted links for items with degree $k$ in the test set.

The item-degree-dependent recall, $R_{k \leqslant 10}$, in the top $L=50$ recommendation list, compared with the HHP method, again presents a significant improvement for all the three datasets, whereas $R_{k>10}$ is a little elevated for the $R Y M$, and stabilized for the Netflix and MovieLens. When compared with the CF method, the OHHP method outperforms in both the $R_{k \leqslant 10}$ and the $R_{k>10}$. It further indicates that the OHHP method prefers to benefit for cold-items recommendation.

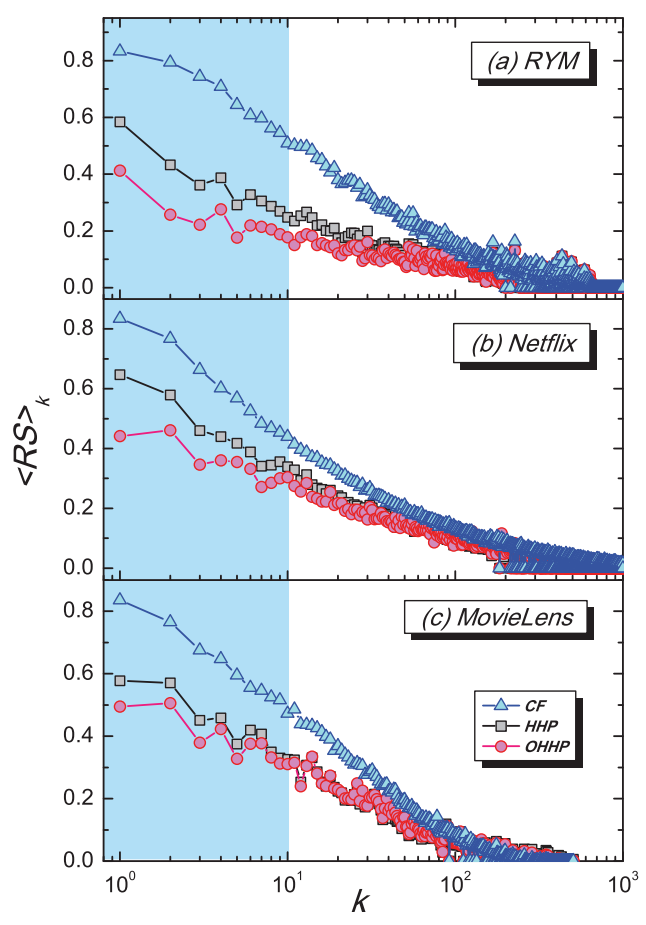

Fig. 4: (Color online) The item-degree-dependent ranking score $\langle R S\rangle_{k}$ vs. the item degree.

Furthermore, compared with the HHP method, the overall precision $P$ in the OHHP method is also found to be enhanced for the RYM, and kept for the Netflix and the MovieLens, while much improved for all the three datasets when compared with the CF method. Again, when we concern particularly on the precision by an itemdegree-dependent precision defined as $P_{k}=\frac{1}{m} \frac{\sum_{i}^{m} q_{i L}^{k}}{L}$, where $q_{i L}^{k}$ is the number of user $u_{i}$ 's deleted links for items with degree $k$ in the top $L$ recommended items list. Similarly, the OHHP method outperforms the CF method in both the $P_{k \leqslant 10}$ and the $P_{k>10}$ in the top $L=50$ recommendation list, and outperforms the HHP method in the $P_{k \leqslant 10}$ for all the three datasets, whereas it presents a little improvement for the $R Y M$, and is statistically steady for the Netflix and the MovieLens in the precision $P_{k>10}$. 


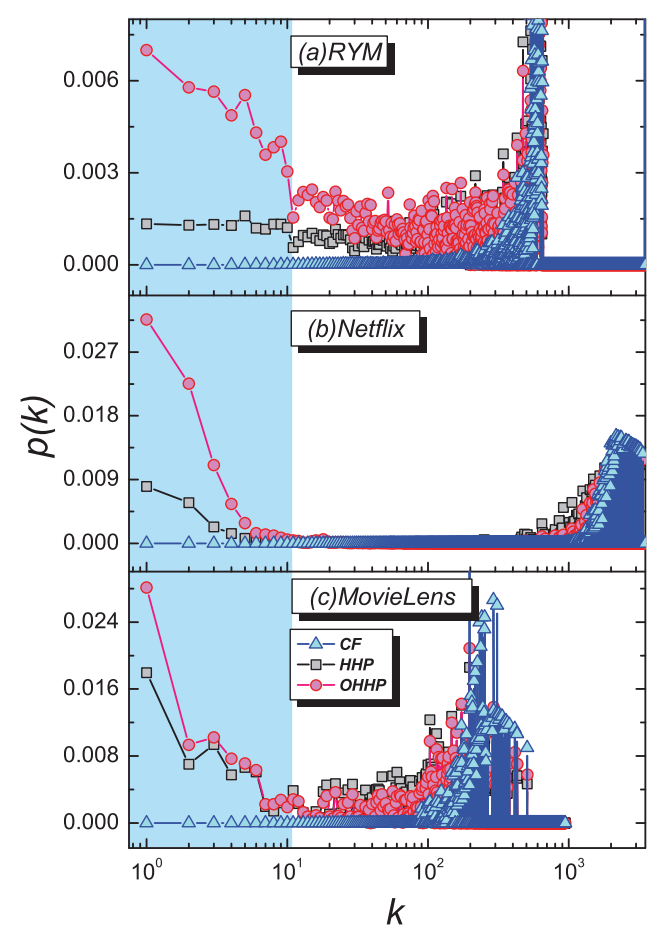

Fig. 5: (Color online) The degree distribution $p(k)$ for the items in the top $L=50$ recommendation list.

It confirms the previous results of ranking score and recall.

Taken together, the OHHP method can significantly enhance the recommendation accuracy of the cold items, while simultaneously it can improve or stabilize the recommendation accuracy of the overall and popular items.

Conclusions. - In this letter, we proposed a recommendation algorithm by introducing an itemdegree-dependent function to improve the recommendation accuracy. With this function, we assume that different items tend to have different fitting parameters in a hybrid recommender system. Experimental results demonstrated that the proposed algorithm outperforms the baseline method.

One of the central tasks of recommender systems is to accurately recommend the unpopular items with insufficient accessorial information without losing the overall or the popular item recommendation accuracy. In this letter, we have shown how the significant dilemma between the recommendation accuracy of the cold items and the popular or the overall items be solved by introducing an item-degree-dependent parameter into the hybrid method of the heat conduction and the probability spreading (OHHP). Compared with the HHP method, the OHHP method significantly enhances the recommendation accuracy of the cold items while it elevates a little or stabilizes the overall and the popular items recommendation accuracy based on three datasets. In addition, while not requiring any additional information, the OHHP method keeps the simple version as the HHP method by directly making use of the bipartite graph structure. Therefore, the present work might shed some light on better understanding and solving the cold-start problem in recommender systems.

This work is partially supported by the National Natural Science Foundation of China under Grant No. 10805025. Z-KZ and TZ acknowledge the National Natural Science Foundation of China under the Grant Nos. 60973069 and 90924011, and the Sichuan Provincial Science and Technology Department (Grant No. 2010HH0002).

\section{REFERENCES}

[1] Masum H. and Zhang Y.-C., First Monday, 9 (2004) 7.

[2] Adomavicius G. and Tuzhilin A., IEEE Trans. Knowl. Data Eng., 17 (2005) 734.

[3] Schafer J. B., Frankowski D., Herlocker J. and Sen S., Lect. Notes Comput. Sci., 4321 (2007) 291.

[4] Pazzani M. J. and Billsus D., Lect. Notes Comput. Sci., 4321 (2007) 325.

[5] Goldberg K., Roeder T., Gupta D. and Perkins C., Inf. Retr., 4 (2001) 133.

[6] Maslov S. and Zhang Y.-C., Phys. Rev. Lett., 87 (2001) 248701.

[7] Hofmann T., ACM Trans. Inf. Syst., 22 (2004) 89.

[8] Blei D. M., Ng A. Y. and Jordan M. I., J. Mach. Learn. Res., 3 (2003) 993.

[9] Laureti P., Moret L., Zhang Y.-C. and Yu Y.-K., Europhys. Lett., 75 (2006) 1006.

[10] Ren J., Zhou T. and Zhang Y.-C., EPL, 82 (2008) 58007.

[11] Zhang Y.-C., Blattner M. and Yu Y.-K., Phys. Rev. Lett., 99 (2007) 154301.

[12] Zhou T., Ren J., Medo M. and Zhang Y.-C., Phys. Rev. E, 76 (2007) 046115.

[13] Jia C. X., Liu R.-R., Sun D. and Wang B.-H., Physica A, 387 (2008) 5887.

[14] Zhou T., Su R.-Q., Liu R.-R., Jiang L.-L., Wang B.H. and Zhang Y.-C., New J. Phys., 11 (2009) 123008.

[15] Liu J. and Deng G.-S., Physica A, 388 (2009) 3643.

[16] Zhou T., Kuscsik Z., Liu J.-G., Medo M., Wakeling J. R. and Zhang Y.-C., Proc. Natl. Acad. Sci. U.S.A., 107 (2010) 4511.

[17] Liu R.-R., Jia C.-X., Zhou T., Sun D. and Wang B.-H., Physica A, 388 (2009) 462.

[18] Zhang Z.-K., Zhou T. and Zhang Y.-C., Physica A, 389 (2010) 179.

[19] Shang M.-S., Zhang Z.-K., Zhou T. and Zhang Y.-C., Physica A, 389 (2010) 1259.

[20] Ahn H. J., Inf. Sci., 178 (2008) 37.

[21] Zhang Z.-K., Liu C., Zhang Y.-C. and Zhou T., EPL, 92 (2010) 28002.

[22] Herlocker J. L., Konstan J. A., Terveen L. G. and RIEDL J. T., ACM Trans. Inf. Syst., 22 (2004) 5.

[23] Zhou T., Jiang L.-L., Su R.-Q. and Zhang Y.-C., EPL, 81 (2008) 58004. 